\title{
Setting priorities to address cardiovascular diseases through universal health coverage in low- and middle-income countries
}

\author{
David A Watkins, ${ }^{1,2}$ Rachel A Nugent ${ }^{3,4}$
}

\begin{abstract}
${ }^{1}$ Division of General Internal Medicine, University of Washington, Seattle, Washington, USA ${ }^{2}$ Department of Medicine, University of Cape Town, Cape Town, South Africa ${ }^{3}$ Research Triangle Institute International, Research Triangle Park, North Carolina, USA ${ }^{4}$ Department of Global Health, University of Washington, Seattle, Washington, USA
\end{abstract}

\section{Correspondence to} Dr David A Watkins, Division of General Internal Medicine, University of Washington, 325 9th Ave Box 359780, Seattle, WA 98104, USA; davidaw@uw.edu

Received 15 December 2016 Revised 31 January 2017 Accepted 1 February 2017

\section{CrossMark}

To cite: Watkins DA, Nugent RA. Heart Asia 2017:9:54-58.

doi:10.1136/heartasia-2015010690

\section{ABSTRACT}

Over the past decade, universal health coverage (UHC) has emerged as a major policy goal for many low- and middle-income country governments. Yet, despite the high burden of cardiovascular diseases (CVD), relatively little is known about how to address CVD through UHC. This review covers three major topics. First, we define UHC and provide some context for its importance, and then we illustrate its relevance to CVD prevention and treatment. Second, we discuss how countries might select high-priority CVD interventions for a UHC health benefits package drawing on economic evaluation methods. Third, we explore some implementation challenges and identify research gaps that, if addressed, could improve the inclusion of CVD into UHC.

\section{INTRODUCTION}

Although health systems are primarily concerned with improving population health, they have several other important objectives: improving the distribution of health in the population, protecting against the financial risks of illness and delivering healthcare efficiently in a manner that is responsive to the needs and expectations of the population. ${ }^{1}$ In the early 2000s, the 'financial risk protection' (FRP) objective of health systems began to receive attention in reports of catastrophic out-of-pocket (OOP) healthcare payments routinely faced in many low- and middle-income countries (LMICs). ${ }^{2}$

The prospect of a global epidemic of catastrophic and impoverishing medical expenditure added crucial momentum to the universal health coverage (UHC) movement. A foundational principle of UHC is providing affordable health insurance to all, which ideally creates adequate protection from the financial risks of ill health. Support for and adoption of UHC have grown rapidly over the past decade, and UHC has evolved from an aspirational notion-in the vein of Alma-Ata and Primary Health Care-to an integral aspect of the Sustainable Development Goals. ${ }^{3}$

As we detail in this review, cardiovascular diseases (CVD) are a frequent source of financial risk in LMICs. ${ }^{4}$ Household-level efforts to treat these conditions often result in catastrophic spending, since a very high proportion of payment is out of pocket. Since the major CVDs-including ischaemic heart disease, stroke, cardiomyopathy, rheumatic heart disease (RHD) and congenital heart diseaseare already the chief cause of morbidity and mortality in LMICs, we argue that CVD care needs to feature prominently in UHC. ${ }^{5}$ Unfortunately, inequality in CVD outcomes persists in many countries because of poor availability and affordability of primary and specialised health services for CVD. ${ }^{6}$ Yet, the UHC literature to date has focused primarily on health system reform (in general) or on achieving equitable access to specific priority services, such as those for reproductive, maternal and neonatal/child health. ${ }^{78}$

In light of the mismatch between CVD's importance and its relative absence from the UHC literature, this review seeks to summarise the evidence to date on CVD in the context of UHC and provide a framework for setting priorities in LMICs. We take an economic viewpoint on priority setting and UHC, though we acknowledge that priority setting is an ethical and political process as well. ${ }^{9}$ Our review has three major objectives: (1) to define and characterise UHC and illustrate its relevance to CVD; $(2)$ to summarise technical aspects of the priority setting process and (3) discuss implementation challenges and propose a CVD-UHC research agenda informed by current gaps in evidence. We hope this review will assist clinical and public health practitioners working on CVD in LMICs to critically review the literature, interact with local decision-makers and participate in the global UHC discourse.

\section{UNDERSTANDING UHC AND ITS APPLICATION TO CVD}

UHC principles can be traced back to many years. ${ }^{3}$ The principle of a right to a minimum standard of health for all individuals was first enumerated in the 1948 Universal Declaration of Human Rights and in the founding charter of the WHO. The modern notion of UHC was first proposed in a 2005 World Health Assembly resolution. Various technical aspects of UHC were elaborated in the World Health Reports of 2008, 2010 and 2013. ${ }^{3}$ These efforts culminated in Sustainable Development Goal 3, which calls on countries to 'achieve universal health coverage, including financial risk protection'. ${ }^{10}$

\section{Defining and characterising UHC}

The WHO defines UHC by its goal: 'to ensure that all people obtain the health services they need without suffering financial hardship when paying for them'. ${ }^{11}$ UHC has three important functions: (1) improving access to health services (particularly among disadvantaged populations), (2) providing FRP and (3) improving the health of individuals covered. ${ }^{12}$

Countries may choose from a wide range of tools to realise these three functions. For example, 
training health extension workers to deliver care in rural and underserved areas may increase access. Universal public finance may provide FRP by eliminating copayments for hospitalisation. Enforcement of clinical guidelines and checklists may improve quality and patient outcomes. In other words, a variety of interventions can contribute to the process of achieving UHC. In fact, studies have documented a wide heterogeneity in designs and outcomes across UHC reforms in different countries. ${ }^{12}$

Commonly, UHC schemes are characterised according to how -and to what extent-they address the three major dimensions of health financing (figure 1$):^{13}$

1. Who is covered? A UHC policy could aim to cover the full population or perhaps target the highest-need subgroups (eg, low-income households).

2. How much of the cost is covered? The policy will specify the proportion of healthcare costs to be prepaid OOP through risk pooling or other group payment mechanisms.

3. What services are covered? As discussed further below, a 'benefits package' will outline the various health services that are covered, considering local epidemiology and demographics, cost, health impact, feasibility and sustainability.

Quality of care complements these dimensions and is crucial to achieving optimal health outcomes under UHC. In many countries, patients express concern over the quality of public sector services and opt to obtain private services instead, or to go without care. Hence, without investments in quality of care, expansion of eligibility to the whole population may not, in itself, be sufficient to achieve full coverage. ${ }^{14}$

If the aim of UHC is to 'fill up' this cube over time, then the question for policy-makers is how much of each dimension to fill and in which order to fill them. Jamison and colleagues identified four general approaches to UHC: (1) progressive universalism, which covers the entire population and minimises OOP payments, (2) a 'balanced' pathway, which attempts to cover more services but with higher OOP payments, (3) private voluntary insurance, supplemented by public finance for those in need and (4) public finance of catastrophic OOP expenses only. ${ }^{15}$ A recent WHO consultation concluded that progressive universalism is probably the fairest, most effective approach. ${ }^{16}$

\section{Relevance of UHC to CVD care}

Where UHC is absent and healthcare is predominately financed through OOP payments, acute CVD services-because they are especially expensive-produce remarkable negative economic consequences for households. In one study, catastrophic health expenditure occurred in more than half of individuals hospitalised for acute coronary syndrome, stroke, acute heart failure or acute peripheral vascular disease in China, India and Tanzania. A substantial number of individuals hospitalised for CVD also reported borrowing money or selling assets to pay for healthcare, and this practice was most common in India, where health insurance coverage was lowest. ${ }^{4}$

The economics of chronic CVD are also noteworthy and relevant to UHC. Because chronic CVD and its risk factors are often asymptomatic or minimally symptomatic, and because primary and secondary prevention are lifelong interventions, financial barriers (all else being equal) can weigh heavily on the decision to engage in care and adhere to medications. For instance, one study in China found that uninsured individuals were less likely to be aware of their hypertension, to receive medication for it and to demonstrate controlled blood pressure. ${ }^{17}$
Given the need for regular interaction with the health system and potential need for expensive diagnostics and therapeutics, especially for late-stage disease, it is possible that UHC could improve CVD outcomes in two ways. First, UHC may improve access to primary care and preventative services and more effectively manage risk conditions such as hypertension and diabetes, such that, over time, the incidence, severity and (by inference) economic impact of CVD are reduced. Second, UHC may finance CVD care (especially acute, inpatient care) directly in order to provide FRP. A well-designed UHC initiative will likely cover a mix of treatment and prevention services in order both to respond to current severe disease and to reduce future disease incidence and its attendant economic consequences.

\section{PRIORITY CVD INTERVENTIONS TO INCLUDE IN UHC Rationale for a health benefits package}

If UHC is to be the framework for health system reform, then a fundamental question is what CVD services are covered (figure 1). Glassman and colleagues have proposed that a 'health benefits package', or a list of priority services, is an important aspect of implementing UHC. ${ }^{18}$ They note that high-income countries with established health systems have gradually incorporated a large number of interventions over time as budgets have grown in parallel to economic development. However, many LMICs considering UHC are starting from a much less resourced position and cannot fund such a broad portfolio of services; so, explicit processes and criteria are critical for defining which interventions to include. ${ }^{18}$

The health benefits package, and the institutionalisation of priority setting in general, is essential if a progressive universalist approach to UHC is taken. If the whole population is covered, the process of defining what is 'in' UHC becomes a political one, engaging the general public and requiring accountability for delivery. Since progressive universalism assumes that healthcare is fully prepaid, usually by government, the fiscal implications of including an intervention in the benefits package need to be considered. This is especially important in LMICs with small public budgets and weak healthcare systems.

\section{Use of effectiveness and cost-effectiveness data}

Identifying the services that could be covered at a given budget level is a deliberative process that starts with a review of disease epidemiology and evidence on effectiveness and costeffectiveness of a wide range of potential interventions, ideally drawing on local or regional data sources. ${ }^{18}$ An example list of CVD services that are likely to be cost-effective and potentially appropriate in LMICs is provided in table 1. This generic list was produced as part of the Disease Control Priorities, Second Edition (DCP2) and is currently being updated for Disease Control Priorities, Third Edition (DCP3; http://www.dcp-3.org). ${ }^{19}$ Rather than being a one-size-fits-all approach to CVD, such a list could serve as a starting point for local analysis and deliberation in countries seeking to develop or refine their health benefits package. The package suggested here must be adapted to local priorities and capacities. Further, lists of priority services must be continually updated with new evidence and adapted to local epidemiology. For example, primary and secondary prevention of rheumatic heart disease were conspicuously absent from DCP2, but recent evidence suggests these interventions can be very cost-effective in low-resource, endemic settings. ${ }^{20}$

However, comparative cost-effectiveness tables (so-called 'league tables') in themselves are merely one aspect of the priority setting process and can be prone to misinterpretation or 
Figure 1 Three dimensions of financing universal health coverage. Figure adapted by authors from refs. ${ }^{13}$ and. $^{15}$

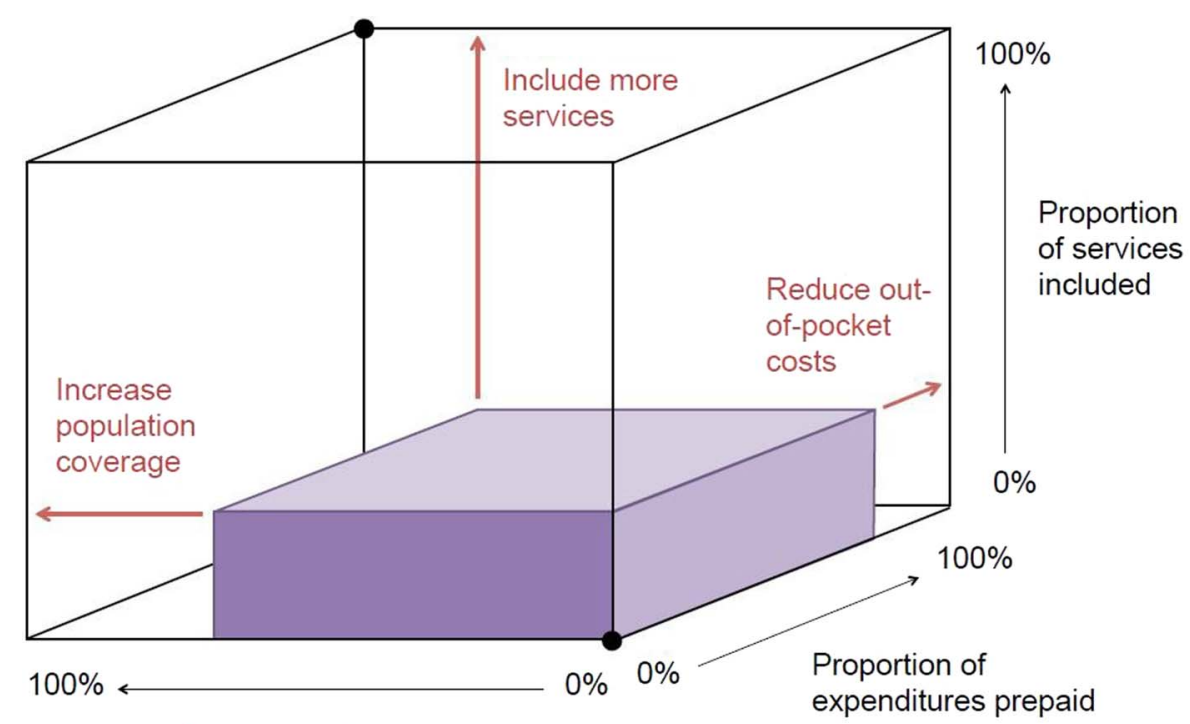

Proportion of population covered

Table 1 Summary of cost-effective clinical services for CVD prevention and treatment in LMICS

\begin{tabular}{|c|c|c|c|}
\hline Condition & Intervention & Objective & $\begin{array}{l}\text { Cost-effectiveness } \\
\text { ratio }\end{array}$ \\
\hline $\begin{array}{l}\text { IHD and stroke } \\
\text { prevention }\end{array}$ & $\begin{array}{l}\text { Combination treatment with aspirin, } \beta \text {-blocker, thiazide diuretic, ACE inhibitor and statin based on } \\
10 \text {-year risk of cardiovascular disease }\end{array}$ & $\begin{array}{l}\text { Primary } \\
\text { prevention }\end{array}$ & US\$2128/DALY averted \\
\hline Tobacco addiction & Nicotine replacement therapy & $\begin{array}{l}\text { Primary } \\
\text { prevention }\end{array}$ & US\$396/DALY averted \\
\hline Acute MI & Aspirin with or without $\beta$-blocker (atenolol) & Acute care & US\$14/DALY averted \\
\hline Acute MI & Incremental use of streptokinase, in addition to aspirin and $\beta$-blocker (atenolol) & Acute care & US\$671/DALY averted \\
\hline $\begin{array}{l}\text { Acute ischaemic } \\
\text { stroke }\end{array}$ & Aspirin dose within 48 hours of onset of acute stroke & Acute care & US\$149/DALY averted \\
\hline $\begin{array}{l}\text { Acute ischaemic } \\
\text { stroke }\end{array}$ & $\begin{array}{l}\text { Heparin within } 48 \text { hours of onset of stroke or thrombolytic therapy using recombinant tissue } \\
\text { plasminogen activator within } 3 \text { hours of onset }\end{array}$ & Acute care & US\$1977/DALY averted \\
\hline $\begin{array}{l}\text { Chronic IHD and } \\
\text { stroke }\end{array}$ & $\begin{array}{l}\text { Combination treatment with aspirin, } \beta \text {-blocker, thiazide diuretic, ACE inhibitor and statin, based on } \\
10 \text {-year risk of cardiovascular disease }\end{array}$ & $\begin{array}{l}\text { Secondary } \\
\text { prevention }\end{array}$ & US\$409/DALY averted \\
\hline Chronic IHD & $\begin{array}{l}\text { Aspirin plus } \beta \text {-blocker (atenolol), with optional ACE inhibitor (enalapril), with or without hospital } \\
\text { availability }\end{array}$ & $\begin{array}{l}\text { Secondary } \\
\text { prevention }\end{array}$ & US\$688/DALY averted \\
\hline Chronic IHD & $\begin{array}{l}\text { Statin (lovastatin), incremental to aspirin, } \beta \text {-blocker (atenolol) and ACE inhibitor (enalapril), with or } \\
\text { without hospital availability }\end{array}$ & $\begin{array}{l}\text { Secondary } \\
\text { prevention }\end{array}$ & US\$2028/DALY averted \\
\hline $\begin{array}{l}\text { Chronic poststroke } \\
\text { care }\end{array}$ & Daily aspirin dose or combination of aspirin and extended release dipyridamole & $\begin{array}{l}\text { Secondary } \\
\text { prevention }\end{array}$ & US\$81/DALY averted \\
\hline $\begin{array}{l}\text { Chronic poststroke } \\
\text { care }\end{array}$ & Carotid endarterectomy surgery & $\begin{array}{l}\text { Secondary } \\
\text { prevention }\end{array}$ & US\$1458/DALY averted \\
\hline Chronic HF & Use of ACE inhibitor and an optional $\beta$-blocker (metoprolol), incremental to diuretics & $\begin{array}{l}\text { Secondary } \\
\text { prevention }\end{array}$ & US\$150/DALY averted \\
\hline
\end{tabular}

misuse. For instance, providing thrombolytics (vs aspirin) for acute ischaemic stroke appears to be cost-effective in Brazil, but it would be inappropriate to make this a priority intervention in a low-income country where the system is not currently able to ensure safe administration (eg, with coagulation studies and neuroimaging capacity). ${ }^{6}$ Further, given the low availability of aspirin in many countries, a more relevant comparison might be aspiring versus current practice-that is, doing nothing. ${ }^{21}$ Finally, significant controversy remains over what costeffectiveness threshold should be used in lower resource settings: recent work suggests that a multiple of 0.5 of per capita gross domestic product (GDP) is more appropriate than the long-standing WHO recommendation of one to three times per capita GDP. ${ }^{22}$

While the findings of DCP2 counter the misconception that CVD care is not cost-effective in LMICs, there are other important considerations for priority setting beyond cost-effectiveness. The most important of these are the related issues of budget impact and optimal resource allocation. Careful budget impact analysis is a critical adjunct to effectiveness and costeffectiveness evidence, since cost-effective interventions may be unaffordable or infeasible if they require large increases in human resources and capital in order to be delivered (so-called 'transition costs'). ${ }^{23}$ An additional analytical step-resource 
allocation across diseases-requires additional modelling in light of budgetary constraints. ${ }^{24}$

\section{Extended cost-effectiveness analysis}

While cost-effectiveness analyses (CEAs) of clinical interventions are highly relevant for developing health benefits packages, CEAs have been critiqued for not considering the other, 'nonhealth' goals of health systems discussed above, and they are most readily applied to assessment of health technologies rather than health policies. To this end, a new method called 'extended cost-effectiveness analysis' (ECEA) was developed for DCP3. ECEA assesses the impact of health policies (such as public finance) and includes two other aspects of health systems: the distributional aspects of the intervention (equity) and the FRP provided. ${ }^{15}$ This method is in harmony with the aforementioned WHO's 'Making Fair Choices' report, which recommended that UHC include priority lists of services (similar to the health benefits package concept) that start with the interventions that are the most cost-effective, prioritise the worst off and -if possible-provide FRP. ${ }^{16}$

To date, three ECEAs have been conducted on CVD policies, including one by Verguet and colleagues that is particularly relevant to UHC. This study compared universal public finance of hypertension care with eight other non-CVD interventions in Ethiopia. For each of the nine interventions, the authors estimated deaths averted per US\$100000 and cases of medical impoverishment averted (ie, by eliminating OOP costs) per US $\$ 100000$ spent on public finance. They found that, while the health impact of hypertension treatment was much lower than, say, childhood vaccines, the FRP was much higher due to the prevention of costly strokes and heart attacks (figure 2). ${ }^{25}$

An unresolved issue in ECEA is how to weight non-health outcomes and compare them with health outcomes as is routinely done in benefit-cost analysis. Such weightings might facilitate intersectoral comparisons-for example, comparing the impact of UHC and the impact of development programmes on poverty alleviation-though these require further empirical research. These limitations aside, ECEA can still provide helpful information about which candidate interventions are likely to provide FRP and are relatively more attractive for inclusion in UHC. As the Ethiopia study demonstrates, ECEAs can make the case for a greater role for CVD prevention and treatment in UHC because of its significant economic benefits.

\section{MOVING FROM EVIDENCE TO POLICY CHANGE Putting CVD on the UHC agenda}

Epidemiological and economic evidence aside, a major barrier to raising the profile of CVD on the UHC agenda is lack of published experience and best practices. The extent to which specific CVD services are included in UHC schemes around the world is not well understood. For instance, the World Bank UHC Study Series details the experience of 24 countries currently implementing UHC reforms. ${ }^{26}$ While most of these countries recognise that CVD is a major problem locally, only six relatively wealthy countries (Brazil, Argentina, Jamaica, Kyrgyz Republic, Chile and China) specifically mention CVD care in their health benefits packages. Advanced CVD services such as cardiac surgery are variably included, for example, in Brazil and Argentina but not Indonesia, suggesting that these ministries have implicitly (or explicitly) adopted rules around what CVD interventions are covered.

Complementing these data, another study found that only $12 \%$ of LMICs listed CVD as a high priority for UHC. ${ }^{27}$ There may be a variety of reasons for this, such as lack of awareness of the importance of CVD, misconception that CVD care is unaffordable or political pressures (domestically, or from foreign donors) to focus on other conditions. Understanding the barriers to getting CVD on the UHC agenda will require further empirical research. Conversely, CVD is beginning to appear in country health strategies in some LMICs that are early adopters of UHC, such as Ethiopia. ${ }^{28}$

It should also be noted that UHC itself is an evolving notion facing numerous implementation barriers in LMICs. A recent case series assessed facilitators to UHC in 11 early adopter countries. ${ }^{9}$ For instance, they noted the importance of advocacy and social movements in adopting UHC reforms. This suggests
Figure 2 Comparison of health and financial risk protection gained per US $\$ 100000$ spent on public finance of selected interventions in Ethiopia. Figure modified from Verguet et $\mathrm{al}_{1}{ }^{25}$ with permission.

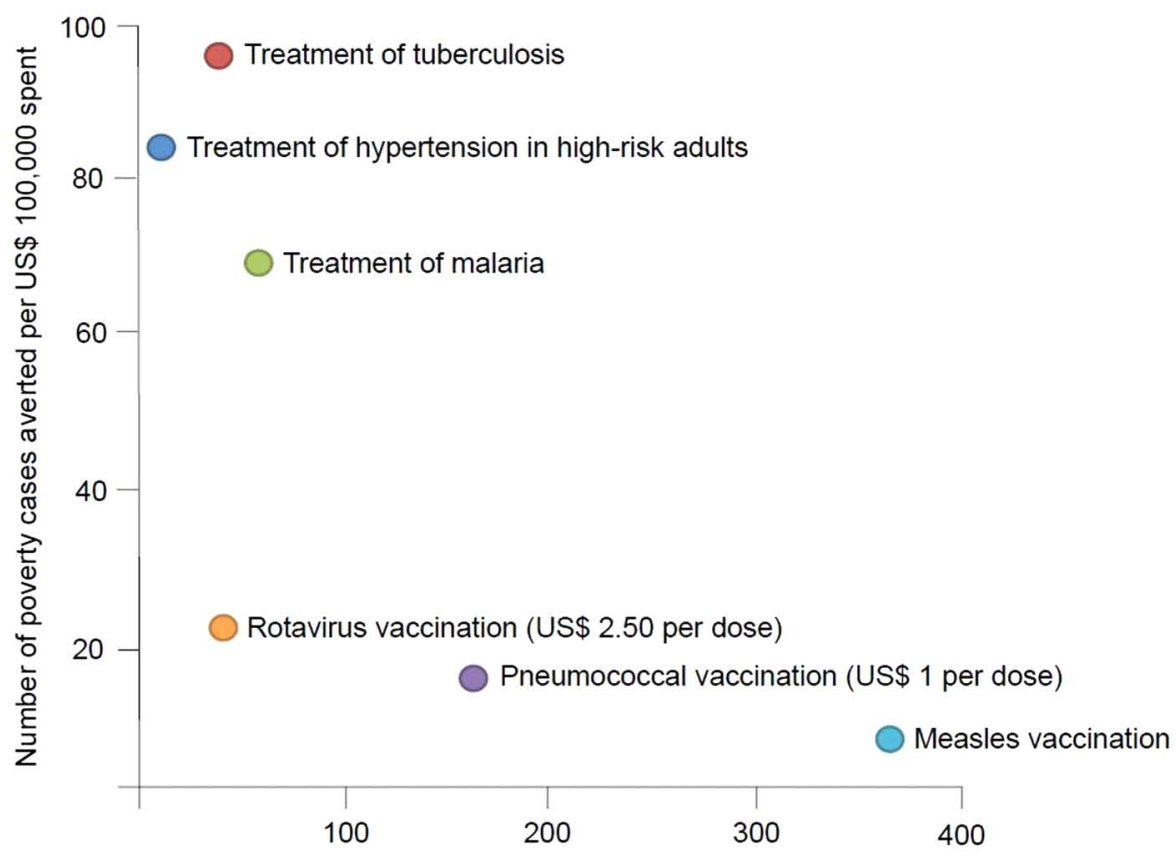

Number of deaths averted per US $\$ 100,000$ spent 
that local CVD practitioners, researchers and patients could engage in the policy process to ensure that CVD is appropriately reflected in the UHC agenda and that local priority setting institutions have sufficient technical expertise to incorporate CVD into their analyses. A second facilitator noted in the report was the ability of countries to address shortfalls in human resources through 'flexible career paths' and 'non-traditional points of entry', particularly in rural and underserved areas. This suggests that the CVD community continue to develop innovative, highquality alternative care delivery platforms (eg, task-sharing through mid-level practitioners and community health workers) that allow for broader population coverage of priority CVD services. ${ }^{29}$

\section{Recommendations for research and policy}

At present, there is still limited information to guide policy around CVD and UHC. More descriptive studies are needed on the health and economic consequences of CVD and the potential effects of public finance on FRP. Longitudinal experimental and quasi-experimental studies are needed in order to understand the effectiveness of new insurance schemes, particularly on chronic diseases whose benefits and costs accrue over time. The existing evidence for the impact of UHC on health has been summarised in a World Bank report, which also highlights the methodological challenges and limitations of the evidence. ${ }^{12}$

The ECEA approach may prove useful for assessing potential ways in which specific CVD policies can contribute to UHC, either directly (by covering treatment) or indirectly (by preventing future disease and its financial consequences). It can make explicit the distributional consequences of policies and tradeoffs between health and FRP. Priority setting institutions could begin to integrate ECEA to provide a richer set of recommendations on the content of health benefits packages. Still, the value of ECEA information will depend on the generation of a large number of new empirical analyses conducted in varying contexts.

Finally, as the number and scope of CVD technologies increase, LMIC governments will be under increased pressure from the private sector to provide these technologies within UHC. This could become a fiscally treacherous and unsustainable path. Countries must find ways to select CVD policies and interventions that most cost-effectively respond to local health needs in a transparent and accountable fashion. ${ }^{18}$ In order to identify and disseminate best practices, what is needed are more reports and case studies of country experiences deliberating and defining the CVD elements of health benefits packages. Research in this area might start with systematic searches of grey literature and government documents from UHC early adopter countries, specifically focusing on CVD services. Best practices, analytical tools and guidelines could then be developed for countries early on the path to UHC and in UHC countries facing a rapidly increasing burden of CVD.

Contributors DAW conceived the study, gathered the literature and wrote the first draft. RAN reviewed the literature and revised the draft for important intellectual content.

Competing interests DAW and RAN are supported by the Disease Control Priorities Network grant from the Bill and Melinda Gates Foundation to the University of Washington. Additionally, DAW is funded by Medtronic Foundation through support to Rheumatic heart disease Evidence-Advocacy-CommunicationHope (RhEACH) and RHD Action, and RAN is funded by RTI International.

Provenance and peer review Commissioned; externally peer reviewed.

\section{REFERENCES}

1 WHO. Everybody business: strengthening health systems to improve health outcomes: WHO's framework for action. Geneva: World Health Organization, 2007.
2 Xu K, Evans DB, Kawabata K, et al. Household catastrophic health expenditure: a multicountry analysis. Lancet 2003;362:111-17.

3 Ji JS, Chen L. UHC presents universal challenges. Health Syst Reform 2016;2:11-14.

4 Huffman MD, Rao KD, Pichon-Riviere A, et al. A cross-sectional study of the microeconomic impact of cardiovascular disease hospitalization in four low- and middle-income countries. PLOS ONE 2011;6:e20821.

5 GBD 2015 DALYs and HALE Collaborators. Global, regional, and national disability-adjusted life years (DALYS) for 315 diseases and injuries and healthy life expectancy (HALE), 1990-2015: a systematic analysis for the Global Burden of Diseases Study 2015. Lancet 2016;388:1603-58.

6 Araújo DV, Teich V, Passos RB, et al. Analysis of the cost-effectiveness of thrombolysis with alteplase in stroke. Arq Bras Cardiol 2010;95:12-20.

7 Yip WCM, Hsiao WC, Chen W, et al. Early appraisal of China's huge and complex health-care reforms. Lancet 2012;379:833-42.

8 Quick J, Jay J, Langer A. Improving women's health through universal health coverage. PLoS Med 2014;11:e1001580.

9 Reich MR, Harris J, Ikegami N, et al. Moving towards universal health coverage: lessons from 11 country studies. Lancet 2016;387:811-16.

10 Sustainable Development Goals. 17 goals to transform our world. Goal 3: ensure healthy lives and promote well-being for all at all ages. United Nations, 2016. http://www.un.org/sustainabledevelopment/health/ (accessed 16 Feb 2016).

11 What is universal health coverage? World Health Organization, 2014. http://www. who.int/features/qa/universal_health_coverage/en/ (accessed 16 Feb 2016).

12 Giedion U, Alfonso EA, Díaz Y. UNICO Studies Series 25. The impact of universal coverage schemes in the developing world: a review of the existing evidence. Washington DC: World Bank, 2013.

13 Busse R, Schreyogg J, Gericke C. Analyzing changes in health financing arrangements in high income countries. HNP discussion paper. Washington DC: World Bank, 2007.

14 Kruk ME. Universal health coverage: a policy whose time has come. BMJ 2013;347: f6360.

15 Jamison DT, Summers LH, Alleyne G, et al. Global health 2035: a world converging within a generation. Lancet 2013;382:1898-955.

16 WHO. Making fair choices on the path to universal health coverage. Final report of the WHO Consultative Group on Equity and Universal Health Coverage. Geneva: World Health Organization, 2014.

17 Feng XL, Pang M, Beard J. Health system strengthening and hypertension awareness, treatment and control: data from the China health and retirement longitudinal study. Bull World Health Organ 2014;92:29-41.

18 Glassman A, Giedion U, Sakuma Y, et al. Defining a health benefits package: what are the necessary processes? Health Syst Reform 2016;2:39-50.

19 Laxminarayan R, Chow J, Shahid-Salles SA. Intervention cost-effectiveness: overview of main messages. In: Jamison DT, Breman JG, Measham AR, et al, eds. Disease control priorities in developing countries. 2nd edn, Chapter 2.

Washington DC: The International Bank for Reconstruction and Development/The World Bank, 2006:60-9.

20 Watkins DA, Lubinga SJ, Mayosi BM, et al. A cost-effectiveness tool to guide the prioritization of interventions for rheumatic fever and rheumatic heart disease control in African nations. PLoS Negl Trop Dis 2016;10:e0004860.

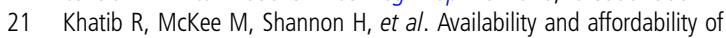
cardiovascular disease medicines and their effect on use in high-income, middle-income, and low-income countries: an analysis of the PURE study data. Lancet 2016;387:61-9.

22 Cairns J. Using cost-effectiveness evidence to inform decisions as to which health services to provide. Health Syst Reform 2016;2:32-8.

23 Hauck K, Thomas R, Smith PC. Departures from cost-effectiveness recommendations: the impact of health system constraints on priority setting. Health Syst Reform 2016;2:61-70.

24 Cleary S, Mooney G, McIntyre D. Equity and efficiency in HIV-treatment in South Africa: the contribution of mathematical programming to priority setting. Health Econ 2010;19:1166-80.

25 Verguet S, Olson ZD, Babigumira JB, et al. Health gains and financial risk protection afforded by public financing of selected interventions in Ethiopia: an extended cost-effectiveness analysis. Lancet Glob Health 2015;3:e288-e96.

26 Universal Health Coverage Study Series (UNICO). 2016. http://www.worldbank.org/ en/topic/health/publication/universal-health-coverage-study-series

27 Gutierrez H, Shewade A, Dai M, et al. Health care coverage decision making in lowand middle-income countries: experiences from 25 coverage schemes. Popul Health Manag 2015;18:265-71.

28 Ministry of Health. Health sector transformation plan, 2015/16-2019/20. Federal Democratic Republic of Ethiopia, 2015.

29 Gaziano TA, Abrahams-Gessel S, Denman CA, et al. An assessment of community health workers' ability to screen for cardiovascular disease risk with a simple, non-invasive risk assessment instrument in Bangladesh, Guatemala, Mexico, and South Africa: an observational study. Lancet Glob Health 2015;3: e556-63. 